\title{
Foley catheter balloon tamponade as a method of controlling iatrogenic pulmonary artery bleeding in redo thoracic surgery
}

\section{Marcus Taylor*, Muhammad Asghar Nawaz, Ozhin Karadakhy, Denish Apparau, Kandadai Rammohan and Paul Waterworth}

Department of Cardiothoracic Surgery, Wythenshawe Hospital, Southmoor Road, Manchester, M23 9LT, UK

\section{Abstract}

Background: Pulmonary artery bleeding secondary to iatrogenic injury is a troublesome intraoperative complication. The likelihood of encountering this complication is significantly higher in redo surgery for a number of reasons, including distortion of anatomical structures, adhesions and loss of tissue planes. Significant blood loss, although rare, remains a concern, and can occasionally be life-threatening. When significant bleeding from the pulmonary artery occurs, it can be a challenging situation to manage.

Case Report: A 65 year old female was undergoing redo thoracic surgery in the setting of a completion lobectomy for biopsy-proven primary adenocarcinoma of the lung. latrogenic injury to the pulmonary artery resulted in significant bleeding that could not be managed by gaining proximal control due to dense adhesions. The novel decision to utilize a Foley catheter for balloon tamponade was taken, in order to provide sufficient haemostasis for definitive surgical repair of the defect to be undertaken

Conclusion: The increased technical difficulties of redo thoracic surgery are well recognised. We describe the first case of Foley catheter balloon tamponade being utilized in the context of iatrogenic pulmonary artery bleeding during thoracic surgery.

\section{Introduction}

Undertaking a pulmonary lobectomy in a virgin chest is recognized as a relatively straightforward operation with acceptably low rates of mortality and morbidity. Redo thoracotomy, particularly in the context of completion lobectomy, represents a more challenging entity. In this situation careful dissection is paramount due to the likelihood of encountering pleuro-parenchymal adhesions, structural architectural changes and loss of tissue planes. These conditions are likely to be even more hazardous if the patient has undergone chemoradiotherapy. The increased risks of bleeding and air leak, as well as the increased operative time, means that redo surgery is usually performed via an open approach, and not via video assisted thoracoscopic surgery (VATS). We describe a case of redo thoracotomy for completion lobectomy where an iatrogenic injury to the pulmonary artery led to significant haemorrhage. The potential need for cardiopulmonary bypass was avoided as the situation was controlled by Foley catheter balloon tamponade.

\section{More Information}

*Address for Correspondence: Marcus Taylor MRCS, Department of Cardiothoracic Surgery, Wythenshawe Hospital, Southmoor Road, Manchester, M23 9LT, UK, Email: marcus.taylor1@nhs.net

Submitted: 23 October 2019

Approved: 19 November 2019 Published: 20 November 2019

How to cite this article: Taylor M, Nawaz MA Karadakhy O, Apparau D, Rammohan K, et al. Foley catheter balloon tamponade as a method of controlling iatrogenic pulmonary artery bleeding in redo thoracic surgery. Arch Case Rep. 2019; 3: 047-049.

DOI: dx.doi.org/10.29328/journal.acr.1001023

Copyright: ( 2019 Taylor M, et al. This is an open access article distributed under the Creative Commons Attribution License, which permits unrestricted use, distribution, and reproduction in any medium, provided the original work is properly cited.

Keywords: Pulmonary artery; latrogenic injury; Foley catheter

Check for updates

\section{Case Description}

A 65-year old morbidly obese female with a raised body mass index (BMI) of $51 \mathrm{~kg} / \mathrm{m}^{2}$ initially underwent a left apical trisegmentectomy for a T2aN0M0 (IASLC $8^{\text {th }}$ edition) primary pulmonary adenocarcinoma of the left upper lobe of the lung. The initial approach was via VATS but the utility port placement confirmed very swiftly that access and visibility were extremely limited despite optimal positioning and downward diaphragmatic traction. Therefore a posterolateral thoracotomy was electively performed. Her co-morbidities included chronic atrial fibrillation, sciatica and chronic obstructive pulmonary disease (COPD). She had a performance status of 1 (PS1) and borderline pulmonary function tests (PFTs) with a Forced Expiratory Volume (FEV1) of $57 \%$ of predicted and diffusing capacity of the lung for transfer of carbon monoxide (DLCO) of $60 \%$ of predicted.

Her case was discussed at the thoracic surgery high 
risk MDT [1], where a parenchymal sparing operation was suggested due to the early stage of the tumour. The patient was counselled about the discussion and informed also that her post-operative pulmonary function would be further reduced (42\% and 44\% predicted for FEV1 and DLCO respectively) if she were to undergo a left upper lobectomy. It was explained that this could further alter her post-operative quality of life. She subsequently underwent an uneventful left thoracotomy and upper lobe trisegmentectomy (S1-S3), from which she made an uncomplicated recovery.

The final histopathological analysis revealed the surprising involvement of station 12 lymph nodes within the parenchyma. Hence the final staging was pT2aN1M0, upstaging her from stage IB to stage IIB, meaning that adjuvant treatment needed to be considered. Following an oncology consultation, the patient declined adjuvant therapy. The subsequent MDT consensus was to offer a completion lobectomy to maximise both disease free and overall survival. The patient was counselled about the risks of redo surgery and chose to pursue this option.

She underwent a redo thoracotomy 16 weeks after her first operation, where, as expected, significant dense adhesions were encountered. During dissection of the hilum the pulmonary artery (PA) was injured, and brisk bleeding ensued. Due to the presence of such dense adhesions, it proved impossible to gain proximal control of the main PA, and the bleeding could only be controlled with direct pressure. A cardiac surgeon was called for assistance, as there were concerns that cardiopulmonary bypass (CPB) would be required in order to achieve haemostasis due to the dense adhesions, the inability to gain proximal control and also the close proximity of the aorta.

It was recognized that attempting to initiate $\mathrm{CPB}$ in this situation would be technically challenging, especially with the patient in the right lateral position, and hence the cardiac surgeon in attendance took the novel decision of employing a Foley catheter in order to achieve endovascular occlusion of the pulmonary artery. This was inserted through the defect in the pulmonary artery and into the lumen. The balloon of the Foley catheter was inflated, achieving satisfactory temporary haemostasis (Image 1). A purse-string 4-0 prolene suture was then stitched around the PA defect. This purse-string was gradually tightened whilst the balloon was deflated, and finally tied off upon removal of the Foley catheter. The final repair of the artery was satisfactory, with good haemostasis achieved.

Due to the unfavourable conditions within the left hemithorax and the extremely high risk of further bleeding, the decision was taken to abandon any further attempts at proceeding to completion lobectomy. Her post-operative stay was otherwise uneventful, and she was discharged home on the $6^{\text {th }}$ post-operative day.

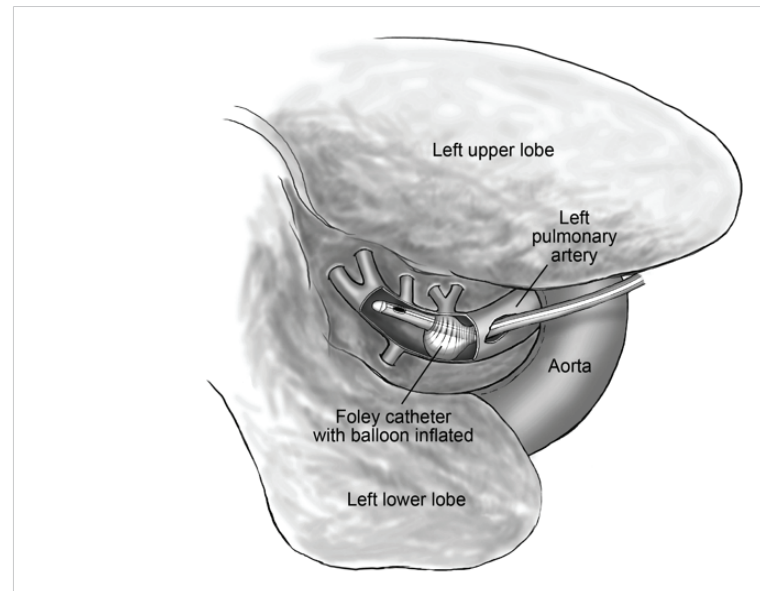

Image 1: Foley catheter inserted through the defect in the artery and inflated.

\section{Discussion}

Pulmonary lobectomy is well recognised as the treatment of choice for early stage lung cancer, where anatomically achievable, for patients possessing enough physiological reserves to withstand the procedure. Whilst mortality after thoracic surgery is low (approximately 1.5\% and 3\% at 30 and 90 days respectively in the UK in 2014), morbidity is often much higher, particularly in the moderate and high-risk patient populations [2]. Risk factors associated with an increased likelihood of developing post-operative complications include advanced age, COPD, raised BMI, smoking status, cardiovascular co-morbidities and pre-operative pulmonary function tests [3].

British Thoracic Society (BTS) guidelines on lung cancer management advocate considering lung parenchyma-sparing surgery in patients with a moderate to high risk of postoperative dyspnea [4]. Whilst preservation of parenchyma where possible is clearly an attractive prospect and has been proven to reduce post-operative dyspnoea (and hence improve quality of life) in comparison to conventional pulmonary lobectomy, this has to be considered alongside the primary aim of lung cancer surgery undertaken with curative intent, which is to provide the optimal oncological resection for the patient, in order to maximize their chances of long term survival.

Redo thoracic surgery is more challenging and associated with a higher rate of complications than first time procedures. This additional complexity is predominately due to the presence of complex intra-thoracic adhesions. An increased risk of bleeding due to damage to the pulmonary vasculature in redo thoracic surgery is well reported in the literature, although very few strategies for management of these potentially perilous complications are mentioned [5]. These complications occur more frequently when performing redo surgery via a VATS approach.

The Foley catheter, whilst primarily utilized for urethral catheterization, has also been described throughout the 
literature as an adjunct for the management of major haemorrhage. The use of the Foley catheter to provide balloon tamponade in the context of acute bleeding was first described in the 1960 s by Taylor \& Williams and has remained a useful weapon in the arsenal of surgeons for the management of major haemorrhage ever since [6]. Whilst there are some reports of Foley catheter balloon tamponade being employed to control iatrogenic bleeding, the literature describes Foley catheter balloon tamponade being employed almost exclusively in the context of trauma, predominately penetrating injuries to the heart and also to vessels of the head \& neck.

When digital pressure is insufficient to control major bleeding and immediate haemorrhage control is required, Foley catheter balloon tamponade can be employed. Without the temporary haemostasis provided by this adjunct one of the only remaining options in this scenario is to attempt to suture the defect. A poorly-visualized operating field (compromised by ongoing substantial bleeding) compounded by the risk of causing further damage (inadvertent damage to coronary arteries or worsening the extent of an injury to an extremely delicate and friable pulmonary artery) means that suturing is rarely a viable option in this salvage scenario and is particularly risky in inexperienced hands. Morbidity and mortality are extremely high in these cases when definitive haemorrhage control is not achieved in the early stages. Multiple case reports and case series detailing penetrating cardiac injuries describe superior outcomes when the management of these patients includes Foley catheter balloon tamponade. Use of the Foley catheter for balloon tamponade has also been described in penetrating neck and maxillofacial injuries and has been shown to be superior to conservative management in this patient population [7-10].

Whilst cardiac trauma and major head \& neck trauma is the main environment in which Foley catheter balloon tamponade is encountered, there are experiences reported of its use in iatrogenic injury. However, we were unable to find any instances of iatrogenic pulmonary artery bleeding controlled in this manner and hence this is the first case report to describe the use of the Foley catheter to control iatrogenic damage to the pulmonary artery.

\section{Conclusion}

The use of the Foley catheter to provide balloon tamponade in severe haemorrhage is well established and well reported. Whilst the majority of these reports refer to its use in the management of trauma, there are also reports of its use in the management of iatrogenic injury. We report for the first time the successful use of Foley catheter balloon tamponade in the management of major thoracic bleeding following iatrogenic pulmonary artery injury in unfavourable operating conditions with limited alternative options. We feel it is important that this approach is recognized as a safe and effective method of dealing with intra-operative bleeding encountered during thoracic surgery.

We received no funding and there is no conflict of interest

\section{References}

1. Nwaejike N, Elbur E, Malagon I, Dodman N, Hewitt K, et al. Is there a role for the high-risk multidisciplinary team meeting in thoracic surgery? Interact Cardiovasc Thorac Surg. 2016; 22: 397-400.

PubMed: https://www.ncbi.nlm.nih.gov/pubmed/26810916

2. Lung cancer clinical outcomes publication 2016. Royal College of Physicians \& Society for Cardiothoracic Surgery in Great Britain \& Ireland.

3. Agostini $\mathrm{P}$, Cieslik $\mathrm{H}$, Rathinam S, Bishay $\mathrm{E}$, Kalkat MS, et al Postoperative pulmonary complications following thoracic surgery: are there any modifiable risk factors? Thorax. 2010; 65: 815-818.

PubMed: https://www.ncbi.nlm.nih.gov/pubmed/20805178

4. Lim E, Baldwin D, Beckles M, Duffy J, Entwisle J, et al. Guidelines on the radical management of patients with lung cancer. British Thoracic Society. 2010

PubMed: https://www.ncbi.nlm.nih.gov/pubmed/20940263

5. Omasa M, Date H, Takamochi K, Suzuki K, Miyata $Y$, et al Completion lobectomy after radical segmentectomy for pulmonary malignancies. Asian Cardiovasc Thorac Ann. 2016; 24: 450-454. PubMed: https://www.ncbi.nlm.nih.gov/pubmed/27207503

6. Taylor $\mathrm{H}$, Williams E. Arteriovenous fistula following disk surgery. $\mathrm{Br}$ J Surgery. 1962; 50: 47-50.

PubMed: https://www.ncbi.nlm.nih.gov/pubmed/13919962 v

7. Elmoghrabi A, Mohamed M, Eggers A, Parmar N, McCann M. More than just a urinary catheter - Haemorrhage control by using a Foley catheter in a penetrating aortic root injury. Trauma Case Rep. 2016; 5: 13-17. PubMed: https://www.ncbi.nlm.nih.gov/pubmed/29942849

8. Feliciano DV, Burch JM, Mattox KL, Bitondo CG, Fields G. Balloon catheter tamponade in cardiovascular wounds. Am J Surg. 1990; 160: 583-587. PubMed: https://www.ncbi.nlm.nih.gov/pubmed/2252117

9. Weppner J. Improved mortality from penetrating neck and maxillofacial trauma using Foley catheter balloon tamponade in combat. J Trauma Acute Care Surg. 2013; 75: 220-224.

PubMed: https://www.ncbi.nlm.nih.gov/pubmed/23823611

10. Navsaria $P$, Thoma M, Nicol A. Foley catheter balloon tamponade for life-threatening hemorrhage in penetrating neck trauma. World J Surg. 2006; 30:1265-1268.

PubMed: https://www.ncbi.nlm.nih.gov/pubmed/16830215 